Revista Brasileira de Agricultura Irrigada v.12, nº.3, p. 2716 - 2724, 2018

ISSN 1982-7679 (On-line)

Fortaleza, CE, INOVAGRI - http://www.inovagri.org.br

DOI: $10.7127 /$ rbai.v12n300892

Protocolo 892.18 - 13/03/2018 Aprovado em 12/06/2018

\title{
PRODUTIVIDADE DE CULTIVARES DE CEBOLA EM DIFERENTES POPULAÇÕES DE PLANTAS EM SEMEADURA DIRETA
}

\author{
Jony Eishi Yuri ${ }^{1 *}$, Geraldo Milanez de Resende ${ }^{1}$, Nivaldo Duarte Costa ${ }^{2}$
}

\begin{abstract}
RESUMO
O uso correto da cultivar aliado ao espaçamento adequado entre plantas promove a máxima eficiência na produtividade total e comercial de bulbo. Com o objetivo de avaliar a produtividade de cultivares de cebola em diferentes espaçamentos entre plantas, foi conduzido um experimento no período de julho a novembro de 2014. O delineamento experimental utilizado foi de blocos ao acaso em esquema fatorial 5 x 4, compreendendo cinco cultivares (Vale Ouro IPA-11, Alfa São Francisco, Franciscana IPA-10, Serena F1 e Atacama F1) e quatro espaçamentos entre plantas $(6,8,10$ e $12 \mathrm{~cm})$ e quatro repetições, empregando-se o programa SISVAR 5.0. As cultivares Franciscana IPA-10 e Alfa São Francisco expressaram maior produtividade comercial em maiores populações de plantas (6 cm entre plantas), enquanto as cultivares Vale Ouro IPA-11, Atacama e Serena alcançaram as maiores produtividades em maior espaçamento entre plantas $(12 \mathrm{~cm})$. Com aumento do espaçamento entre plantas, detectou-se redução na produtividade de bulbos não comerciais (refugos), sendo que o menor valor ocorreu no espaçamento de $12 \mathrm{~cm}$ entre plantas. Nas condições do Submédio do Vale do São Francisco, em semeadura direta, recomenda-se o espaçamento entre plantas de $6 \mathrm{~cm}$ para as cultivares Franciscana IPA-10 e Alfa São Francisco, $9 \mathrm{~cm}$ para a cultivar Serena, $10 \mathrm{~cm}$ para as cultivar Vale Ouro IPA-11 e $12 \mathrm{~cm}$ para a cultivar Atacama.
\end{abstract}

Palavras-chave: Allium серa, competição, densidade de plantio, rendimento.

\section{YIELD ONION CULTIVARS IN DIFFERENT PLANT POPULATIONS IN DIRECT SOWING}

\begin{abstract}
The correct use of farming combined with the adequate spacing between plants promotes maximum efficiency in yield and bulb quality. Aiming to evaluate the yield of onion cultivars in different spacing between plants, an experiment was conducted in the period from July to November 2014. The experimental design was randomized blocks in a factorial 5 x 4, with five cultivars (Vale Ouro IPA-11, Alfa São Francisco, Franciscana IPA-10, Serena F1 e

\footnotetext{
${ }^{1}$ Autor para correspondência Eng. Agrônomo, D.Sc., Embrapa - Centro de Pesquisa Agropecuária do Trópico Semiárido. E-mails: jony.yuri@embrapa.br ; geraldo.milanez@embrapa.br

${ }^{2}$ Eng. Agrônomo, M.Sc., Embrapa - Centro de Pesquisa Agropecuária do Trópico Semiárido. E-mail:

nivaldo.costa@embrapa.br
} 
Atacama F1) and plant spacing (6, 8, 10 and $12 \mathrm{~cm}$ between plants) and four replications, using the SISVAR 5.0 program. The cultivar Franciscana IPA-10 and Alfa São Francisco showed greater yield in larger populations of plants ( $6 \mathrm{~cm}$ between plants), while the cultivar Vale Ouro IPA-11, Atacama and Serena reached the highest yield in most plant spacing (12 $\mathrm{cm})$. With increased spacing between plants, was detected linear yield reduction bulbs not commercial (Calls) with lower value spacing of $12 \mathrm{~cm}$ between plants (2.0 t ha-1). In the SubMidlle of San Francisco Valley conditions, in direct sowing, the recommended plant spacing of $6 \mathrm{~cm}$ for Franciscana IPA-10 Franciscan and Alfa São Francisco cultivars, $9 \mathrm{~cm}$ to cultivar Serena, and $12 \mathrm{~cm}$ for the cultivars Vale Ouro IPA-11and Atacama.

Keywords: Allium серa, adaptation, planting density, yield.

\section{INTRODUÇÃO}

A cebola (Allium cepa) ocupa entre as hortaliças de maior importância econômica nacional o terceiro lugar. Segundo dados do IBGE (2017), em 2016 a produtividade média ficou em torno de 29,6 t ha ${ }^{-1}$, sendo os estados de Pernambuco e Bahia os maiores produtores do Nordeste com produtividade média de 24,9 e 30,6 t ha ${ }^{-1}$, respectivamente.

O desempenho agronômico de uma dada espécie está relacionado tanto à sua adaptação local, quanto às práticas de manejo fitotécnico (MENEZES JÚNIOR; VIEIRA NETO, 2012). O uso de cultivares superiores e de técnicas modernas de produção como irrigação, alta densidade populacional, semeadura direta, mecanização da produção, adubação balanceada, entre outras, associadas ao uso de sementes de melhor padrão genético vêm favorecendo aumentos gradativos e constantes no rendimento. Alta tecnologia e o uso de cultivares híbridas têm sido fatores de incremento de produtividade, especialmente nas regiões Sudeste e Centro-Oeste e em parte do Nordeste nos últimos anos (LEITE et al., 2009).

Resende et al. (2005b) avaliando cultivares de cebola no Submédio do Vale do São Francisco obtiveram produtividades totais de bulbos entre 19,1 a 45,1 $\mathrm{t} \mathrm{ha}^{-1}$ entre diferentes genótipos, e produtividade comercial oscilando entre 15,6 a 43,1 t ha-1. Já Costa et al. (2007) relatam produtividade total oscilando entre 30,4 a 58,4 t ha ${ }^{-1}$ e comercial entre 24,0 a 56,0 $\mathrm{t} \mathrm{ha}^{-1}$, com massa fresca do bulbo entre 86 e $160 \mathrm{~g}$ bulbo $^{-1}$ para diferentes genótipos. Sousa et al. (2008) afirmam que as cultivares das séries IPA recomendadas para a região Nordeste, atinge bons níveis de produtividade e nas regiões de Mossoró, RN e Petrolina, PE, obtiveram 45,9 e 48,2 t ha ${ }^{-1}$, respectivamente.

Em semeadura direta Rebouças et al. (2008) verificaram que a densidade afetou significativamente a produtividade comercial e massa fresca dos bulbos. O híbrido Mercedes apresentou produtividade total e comercial significativamente superior à cultivar Serrana. A produtividade comercial foi reduzida com o incremento da densidade, e produtividade superior foi obtida no espaçamento de $20 \times 7 \mathrm{~cm}$.

Siqueira (2004) verificou redução na produtividade comercial de bulbos de cebola com a elevação da população de plantas de 714.285 para 1.071.428 plantas $\mathrm{ha}^{-1}$, apresentando valores de 31 e $26 \mathrm{t} \mathrm{ha}^{-1}$, respectivamente, devido redução da massa fresca do bulbo com a elevação do número de plantas por área.

No sistema de transplantio por mudas diferentes autores relataram que em geral o aumento na densidade de plantas proporcionou maior produtividade total, menor produtividade comercial e menor massa de bulbos (RESENDE; COSTA, 2005a; BAIER et al., 2009; MENEZES JÚNIOR; VIEIRA NETO, 2012; HENRIQUES et al., 2014). Entretanto, Geremew et al. (2010) relataram que as cultivares respondem diferentemente ao espaçamento entre plantas. Para as cultivares Nasik Red e Adama Red maiores rendimentos comerciais e menores produções de bulbos não comerciais (refugos) foram obtidos no 
espaçamento de $4 \mathrm{~cm}$ entre plantas, enquanto o espaçamento de $6 \mathrm{~cm}$ entre plantas foi o que obteve melhor resposta para a cultivar Bombay Red.

Assim, o presente trabalho objetivou avaliar cultivares de cebola em diferentes espaçamentos entre plantas, em semeadura direta, visando indicar melhor (es) espaçamento (s) que promovam maiores produtividades e qualidade de bulbo nas condições do Submédio do Vale do São Francisco.

\section{MATERIAL E MÉTODOS}

O experimento foi conduzido no período de julho a novembro de 2014, no Campo Experimental de Bebedouro da Embrapa Semiárido, Petrolina-PE (9 $9^{\prime} \mathrm{S}$, $40^{\circ} 29^{\prime \prime} \mathrm{W}, 365,5 \mathrm{~m}$ de altitude). Segundo a classificação climática de Köppen, a região apresenta clima do tipo BSWh', semiárido. A temperatura média do ar varia de 24,1 a 28,0 ${ }^{\circ} \mathrm{C}$, com as temperaturas máxima e mínima oscilando entre 29,6 a $34,0{ }^{\circ} \mathrm{C}$ e de 18,2 a $22,1^{\circ} \mathrm{C}$, respectivamente. $\mathrm{O}$ período chuvoso concentra-se entre os meses de novembro a abril, com $90 \%$ dos totais anuais, sendo que os meses de janeiro a abril contribuem com $70 \%$ do total anual, destacando-se o mês de março e o de agosto como o mais e o menos chuvoso. A precipitação pluviométrica média anual é de 549 mm (TEIXEIRA, 2010).

O delineamento experimental utilizado foi de blocos ao acaso em esquema fatorial 5 $\mathrm{x} 4$, compreendendo cinco cultivares (Vale Ouro IPA-11, Alfa São Francisco, Franciscana IPA-10, Serena F1 e Atacama F1) e quatro espaçamentos entre plantas $(6,8$, 10 e $12 \mathrm{~cm})$ e quatro repetições. O espaçamento entre linhas manteve-se fixo em $10 \mathrm{~cm}$ e totalizou populações de 520.833 (10 x $12 \mathrm{~cm}), 625.000$ (10 x $10 \mathrm{~cm}), 781.250$ (10 x $8 \mathrm{~cm})$ e 1.041 .666 plantas ha-1 $(10$ x $6 \mathrm{~cm})$.

A semeadura foi realizada diretamente no local definitivo utilizando-se uma calha de tubo PVC azul de uma polegada com furos espaçados conforme os espaçamentos entre plantas previamente estabelecidos para facilitar a distribuição das sementes. As parcelas constaram de canteiros com 1,6 m de largura por 1,2 m de comprimento (1,92 $\left.\mathrm{m}^{2}\right)$.

As irrigações foram feitas através do método de gotejamento utilizando-se fitas gotejadoras com emissores espaçados de 0,30 m e vazão de 1,2 $\mathrm{L} \mathrm{hora}^{-1}$, com turno de rega diário, com lâminas em torno de $5 \mathrm{~mm}$. A adubação de plantio de acordo com a análise de solo $\left(\mathrm{pH}\left(\mathrm{H}_{2} \mathrm{O}\right)=6,0 ; \mathrm{Ca}=18 \mathrm{mmol}_{\mathrm{c}} \mathrm{dm}^{-3}\right.$; $\mathrm{Mg}=6$ mmolc $_{\mathrm{c}} \mathrm{dm}^{-3} ; \mathrm{Na}=0,1 \mathrm{mmol}_{\mathrm{c}} \mathrm{dm}^{-3} ; \mathrm{K}$ $=4,1 \mathrm{mmol}_{\mathrm{c}} \mathrm{dm}^{-3} ; \mathrm{Al}=0,0 \mathrm{mmol}_{\mathrm{c}} \mathrm{dm}^{-3}, \mathrm{P}$ $\left(\right.$ Mehlich) $=40,0 \mathrm{mg} \mathrm{dm}^{-3}$ e M.O. $=3,0 \mathrm{~g} \mathrm{~kg}^{-1}$ ) constou de $600 \mathrm{~kg} \mathrm{ha}^{-1}$ da fórmula 06-24-12 e em cobertura via água de irrigação, $90 \mathrm{~kg} \mathrm{ha}^{-1}$ de $\mathrm{N}$ (ureia), $100 \mathrm{~kg} \mathrm{ha}^{-1}$ de $\mathrm{P}_{2} \mathrm{O}_{5}$ (MAP), 90 $\mathrm{kg} \mathrm{ha}^{-1}$ de $\mathrm{K}_{2} \mathrm{O}$ (nitrato de potássio), $60 \mathrm{~kg} \mathrm{ha}^{-}$ ${ }^{1}$ de Ca (nitrato de cálcio) e $60 \mathrm{~kg} \mathrm{ha}^{-1}$ de $\mathrm{Mg}$ (sulfato de magnésio) até 70 dias após o transplantio. Os tratos culturais e fitossanitários foram os comuns a cultura da cebola.

A colheita foi realizada quando em média $90 \%$ das plantas encontravam-se tombadas (estalo), conforme o ponto de colheita de cada cultivar. As plantas colhidas foram submetidas ao processo de cura, ficando por três dias expostas ao sol e cinco dias à sombra, efetuando-se, em seguida, o corte da parte aérea.

Avaliou-se o ciclo vegetativo, número de bulbos comerciais por parcela, a produtividade total, comercial (bulbos perfeitos e com diâmetro transversal acima de $35 \mathrm{~mm}$ ) e não comercial de bulbos (refugos) expressas em $t \mathrm{ha}^{-1}$. A massa fresca de bulbo (g bulbo-1) foi determinada dividindo-se 0 peso de bulbos comerciais após a cura pelo número de bulbos. Os dados coletados foram submetidos à análise de variância e seus efeitos avaliados pelo teste de $\mathrm{F}$ a $5 \%$ de probabilidade. Utilizou-se regressão para as médias de espaçamentos entre plantas e teste de Tukey a 5\% de probabilidade para cultivares, empregando-se o programa SISVAR 5.0 (FERREIRA, 2011).

\section{RESULTADOS E DISCUSSÃO}


A cultivar Atacama apresentou o menor ciclo vegetativo com 116 dias, seguida pela cultivar Franciscana IPA-10 com 120 dias, sendo que as demais cultivares apresentaram ciclo de 122 dias, independentemente dos espaçamentos usados. Segundo Souza; Resende (2002) cultivares com ciclo de 4 a 5 meses são caracterizadas como precoces, ou seja, de ciclo curto, necessitando de fotoperíodo de 10 a 11 horas para bulbificação.

A análise de variância revelou efeito de interação entre o espaçamento de plantas versus cultivares para as variáveis produtividade total, comercial, número de bulbos comerciais e massa fresca de bulbo.

O aumento da população de plantas reduziu, de forma linear a produtividade total de bulbos (Figura 1) na cultivar Vale Ouro IPA-11 e Atacama, que obtiveram valores similares, onde o maior espaçamento entre plantas $(12 \mathrm{~cm})$ proporcionou incrementos da ordem de 64,0 e 63,5\% superiores, quando comparado ao menor espaçamento, $6 \mathrm{~cm}$

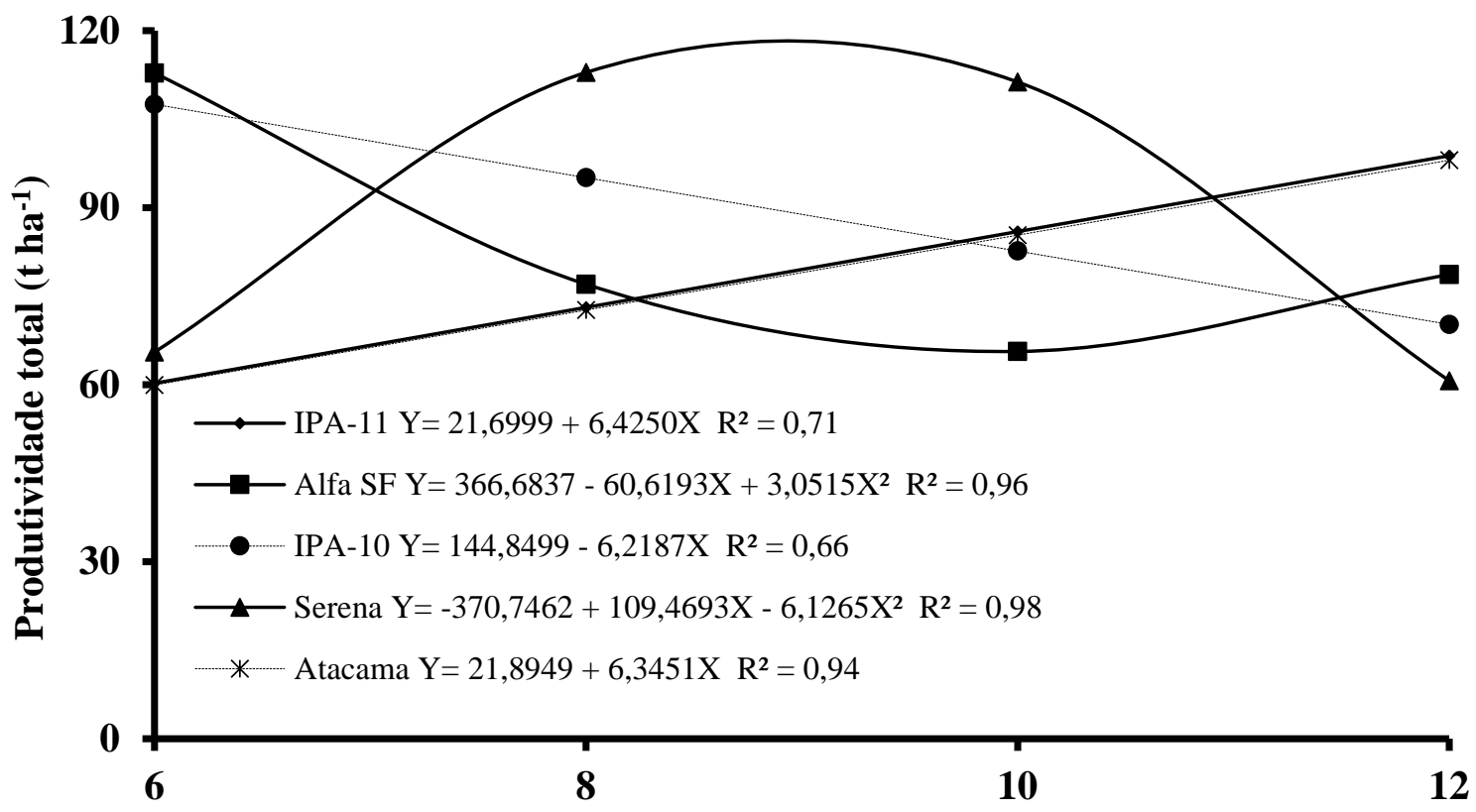

Espaçamentos entre plantas (cm)

Figura 1. Produtividade total de bulbos de cultivares de cebola em função de diferentes espaçamentos entre plantas. Petrolina-PE, 2014.

Enquanto a cultivar Franciscana IPA-10 evidenciou efeito linear negativo, ou seja, o menor espaçamento de $6 \mathrm{~cm}$ entre plantas alcançou rendimento superior em 53,1\% comparativamente ao espaçamento de $12 \mathrm{~cm}$, demonstrando esta cultivar um melhor arranjo populacional quando em plantio adensado. Efeito quadrático com ponto de mínima produtividade foi observado para a cultivar Alfa São Francisco, atingindo o menor valor no espaçamento de $10 \mathrm{~cm}$ entre plantas, com maior rendimento verificado no menor espaçamento de $6 \mathrm{~cm}\left(112,8 \mathrm{t} \mathrm{ha}^{-1}\right)$. Resultado similar, no entanto, com ponto de máxima produtividade foi verificado para a cultivar Serena, que obteve o maior rendimento no espaçamento de $9 \mathrm{~cm}$ entre plantas $\left(118,2 \quad \mathrm{t} \quad \mathrm{ha}^{-1}\right) . \quad$ Os resultados observados demonstram um comportamento distinto entre cultivares ao efeito da do adensamento de plantas, concordando com Geremew et al. (2010), que informaram respostas diferenciadas das cultivares quando submetidas a diferentes espaçamentos entre plantas. Kahsay et al. (2014) obtiveram maior produtividade total de bulbos no espaçamento de $5 \mathrm{~cm}$ comparativamente ao maior de $10 \mathrm{~cm}$ entre plantas, enquanto Baier et al. (2009) observaram que a 
produtividade total de bulbos aumentou linearmente em função do aumento na densidade de plantas.

Resultados similares foram obtidos para produtividade comercial de bulbos (Figura 2). O aumento da população de plantas reduziu, de forma linear a produtividade de bulbos nas cultivares Vale Ouro IPA-11 e Atacama, onde o maior espaçamento entre plantas $(12 \mathrm{~cm})$ proporcionou incrementos da ordem de 75,3 e $66,7 \%$ superiores ao menor espaçamento de 6 $\mathrm{cm}$.

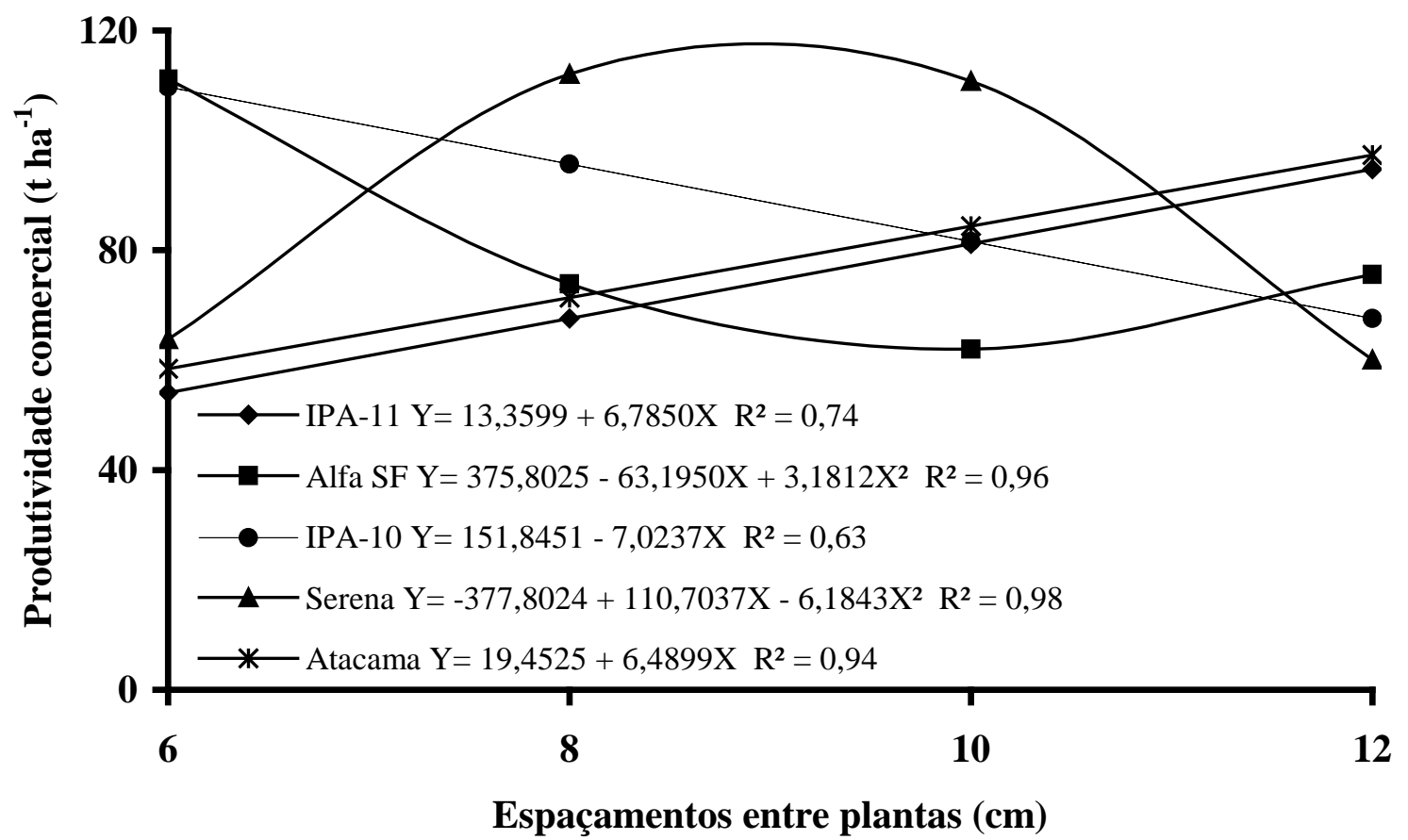

Figura 2. Produtividade comercial de bulbos de cultivares de cebola em função de diferentes espaçamentos entre plantas. Petrolina-PE, 2014.

A cultivar Franciscana IPA-10 evidenciou efeito linear negativo, onde o menor espaçamento de $6 \mathrm{~cm}$ entre plantas alcançou rendimento superior em $62,4 \%$, comparativamente ao espaçamento de $12 \mathrm{~cm}$, demonstrando esta cultivar uma melhor resposta quando se aumenta o número de plantas por área. Efeito quadrático com ponto de mínima produtividade foi observado para a cultivar Alfa São Francisco, atingindo o menor valor no espaçamento de $9,9 \mathrm{~cm}$ entre plantas $\left(62,1 \mathrm{t} \mathrm{ha}^{-1}\right)$, com maior rendimento verificado no menor espaçamento de $6 \mathrm{~cm}$ $\left(111,2 \mathrm{t} \mathrm{ha}^{-1}\right)$. Resultados similares, no entanto, com ponto de máxima produtividade foram verificados para a cultivar Serena que obteve o maior rendimento no espaçamento de $8,9 \mathrm{~cm}$ entre plantas $\left(117,6 \mathrm{t} \mathrm{ha}^{-1}\right)$. Os resultados demonstram em relação ao arranjo populacional comportamento diferenciado de resposta entre as cultivares. As cultivares
Franciscana IPA-10 e Alfa São Francisco expressaram maior produtividade em maiores populações de plantas (6 cm entre plantas), enquanto as cultivares Vale Ouro IPA-11, Atacama e Serena alcançaram as maiores produtividades em maior espaçamento entre plantas (9 a $12 \mathrm{~cm})$. Entre as cultivares de polinização aberta a cultivar Alfa São Francisco foi a mais produtiva, e entre os híbridos "Serena" foi superior. As produtividades obtidas nesse trabalho foram bastante superiores às médias brasileira, pernambucana e baiana na safra de 2014 (IBGE, 2015). Híbridos com produtividades acima de 80,0 $\mathrm{t} \mathrm{ha}^{-1}$ como no presente estudo são informados por Quartiero et al. (2014). Bandeira et al. (2013) recomendaram o híbrido Serena como o mais produtivo, comparativamente as cultivares locais Alfa São Francisco, Vale Ouro IPA-11, Franciscana IPA-10. Em geral, o incremento 
na população de plantas promove aumento na produtividade total e redução na comercial, em função da menor massa fresca de bulbos, resultados estes encontrados por Siqueira (2004). No entanto, afirmaram Geremew et al. (2010) que as cultivares respondem diferentemente ao espaçamento entre plantas, em um mesmo local de cultivo, fato esse constatado no presente trabalho.

Não se verificou interação entre espaçamentos entre plantas e cultivares para produtividade de bulbos considerados não comerciais (refugos) os quais foram influenciados significativamente de forma independente. Com aumento do espaçamento entre plantas, se detectou redução linear ( $\mathrm{Y}=$ $\left.3,4680-0,1157 \mathrm{X} \mathrm{R}^{2}=0,95\right)$ com $\mathrm{o}$ incremento dos espaçamentos entre plantas, com menor valor no espaçamento de $12 \mathrm{~cm}$ $\left(2,0 \mathrm{t} \mathrm{ha}^{-1}\right)$. Estes resultados mostram relação inversa entre o aumento do espaçamento entre plantas e a produção de bulbos não comerciais. Para cultivares se verificou uma variação entre $0,90 \mathrm{t} \mathrm{ha}^{-1}$ (cultivar Serena) e $5,3 \mathrm{t} \mathrm{ha}^{-1}$ (Vale Ouro IPA-11), as demais se situaram entre 1,1 e 2,8 t ha ${ }^{-1}$. Resende; Costa (2005a) e Henriques et al. (2014) também observaram com o aumento da densidade populacional de plantas maior produtividade de bulbos não comerciais.

O espaçamento entre plantas promoveu efeitos significativos nas cultivares para número de bulbos comerciais por parcela (Figura 3), à exceção da cultivar Atacama, onde não se observou diferenças com variação entre $93(6 \mathrm{~cm})$ a 98 bulbos $(12 \mathrm{~cm})$.

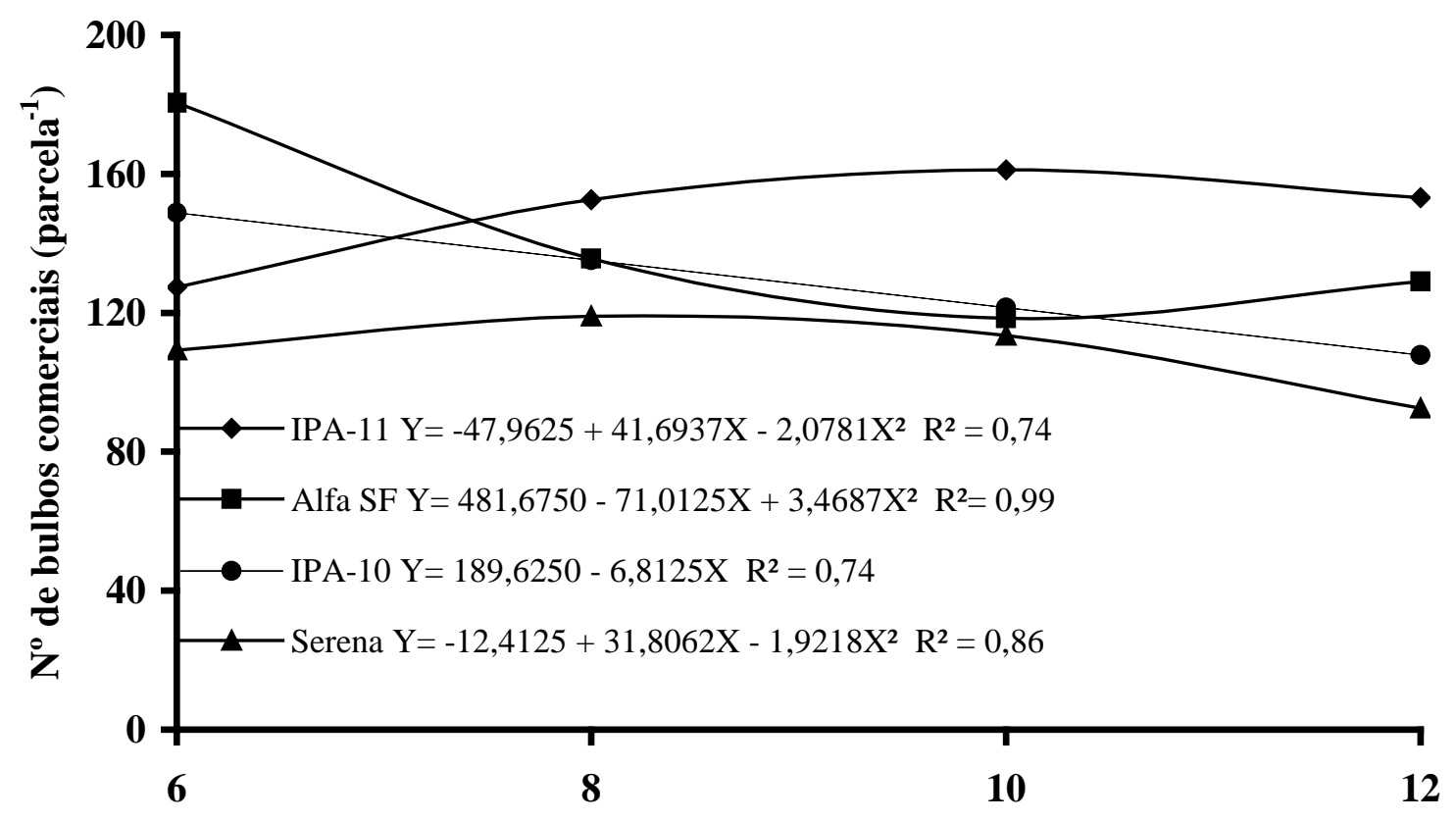

Espaçamentos entre plantas (cm)

Figura 3. Número de bulbos comerciais por parcela de cultivares de cebola em função de diferentes espacamentos entre plantas. Petrolina-PE. 2014.

Efeito linear negativo com o incremento dos espaçamentos entre plantas foi obtido pela cultivar Franciscana IPA-10 onde o menor espaçamento entre plantas de $6 \mathrm{~cm}$ apresentou o maior número (149 bulbos) comparativamente ao espaçamento de $12 \mathrm{~cm}$ (108 bulbos). Desdobrando-se a equação quadrática verifica-se que os espaçamentos entre plantas de 8,3 e 10,0 cm foram os que obtiveram os maiores números de bulbos comerciais para as cultivares Serena e Vale Ouro IPA-11. Entretanto, para a cultivar Alfa São Francisco o espaçamento de $10,2 \mathrm{~cm}$ foi o que demonstrou ser o menos recomendado pelo menor número de bulbos comerciais produzidos (118,2 bulbos) comparativamente ao espaçamento de $6 \mathrm{~cm}$ que alcançou 180,5 bulbos. O que se observa, assim com para 
produtividade é um comportamento diferenciado em termos responsivos tanto para cultivares como híbridos de cebola, o que concorda com as afirmações de Geremew et al. (2010) e Kahsay et al. (2014) que observaram maior número de bulbos comerciais com o aumento do espaçamento entre plantas.

Com exceção da cultivar Atacama que evidenciou efeito linear positivo com 0 incremento dos espaçamentos entre linhas, onde o espaçamento de $12 \mathrm{~cm}$ com 185,7 g bulbo $^{-1}$ apresentou a maior massa, os demais tratamentos demonstraram efeito quadrático com ponto de mínima ou máxima massa fresca de bulbo comercial (Figura 4). Os espaçamentos entre linha que apresentaram as menores massas frescas de bulbos foram de 7,8 cm para a cultivar Vale Ouro IPA-11, 9,8 cm para "Alfa São Francisco" e 10,2 cm para Franciscana IPA-10. Os melhores resultados de massa fresca foram de 12 para Vale Outo
IPA-11 e de $6 \mathrm{~cm}$ para Franciscana IPA-10 e Alfa São Francisco. Para a cultivar Serena observou-se ponto de máxima massa no espaçamento 9,1 cm com 184,4 g bulbo-1. Gashua; Abbator (2013), Kahsay et al. (2014) e Bosekeng; Coetzer (2015) relataram redução na massa fresca de bulbo com o aumento da densidade de plantio. Assim como as demais variáveis avaliadas o comportamento das cultivares em termos de massa fresca foi bem particularizado, específico, o que é justificado pela sua adaptação as condições locais de cultivo, e, sobretudo, pelas características suas genéticas. Em termos médios, as cultivares apresentaram valores distintos de massa fresca comercial de bulbo. A menor massa média foi verificada para a cultivar Vale Ouro IPA-11 (96 g bulbo ${ }^{1}$ ), seguida pelas cultivares Alfa São Francisco (106 g bulbo-1), Franciscana IPA-10 (129 g bulbo $\left.^{-1}\right)$, Serena (144 g bulbo ${ }^{-1}$ ) e Atacama (152 bulbo $^{-1}$.

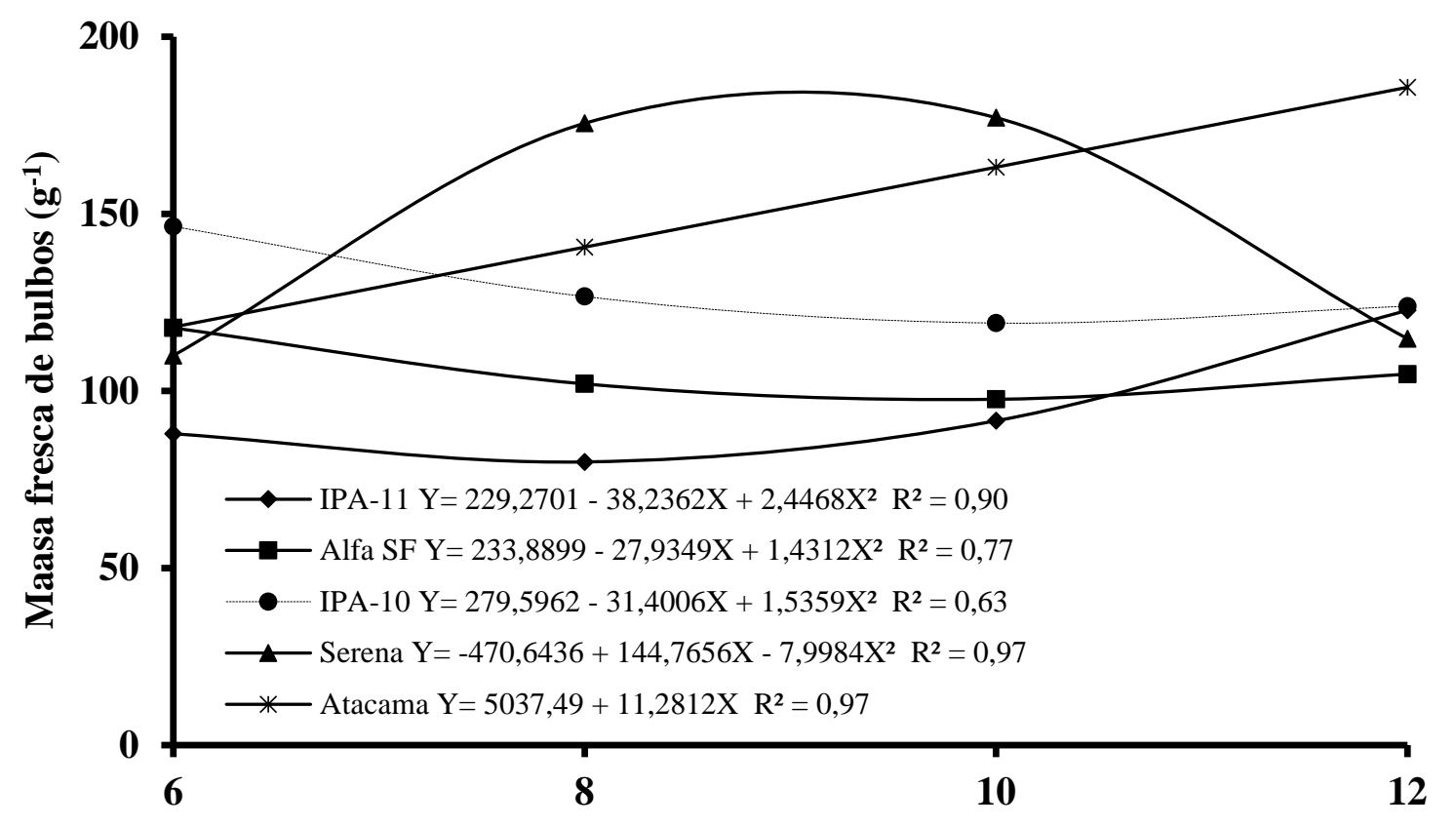

Espaçamentos entre plantas (cm)

Figura 4. Massa fresca de bulbos de cultivares de cebola em função de diferentes espaçamentos entre plantas. Petrolina-PE, 2014.

Na década de 90, os bulbos com massa fresca entre 80 e $100 \mathrm{~g}$ tinham a preferência do consumidor (SILVA et al., 1991). Com o desenvolvimento de novas cultivares, sobretudo, as híbridas, que apresentam além de maior produtividade, uniformidade, formato, compacidade (bulbos de maior massa fresca em relação ao diâmetro) e maior conservação pós-colheita, já verifica-se aceitação de bulbos de tamanho 
superior. Na prática, bulbos entre 80 a 180 g seriam os mais adequados como condimento, nesse contexto todas as cultivares avaliadas atendem plenamente as exigências do mercado consumidor.

\section{CONCLUSÕES}

Com aumento do espaçamento entre plantas verificou-se redução na produtividade de bulbos não comerciais (refugos), com menor valor obtido no espaçamento de $12 \mathrm{~cm}$ entre plantas.

Maiores produtividades comerciais, em semeadura direta, nas condições do Submédio do Vale do São Francisco são alcançadas no espaçamento entre plantas de $6 \mathrm{~cm}$ para as cultivares Franciscana IPA-10 e Alfa São Francisco, $9 \mathrm{~cm}$ para a cultivar Serena, e 10 cm para as cultivar Vale Ouro IPA-11 e 12 cm para cultivar Atacama.

\section{REFERÊNCIAS}

BAIER, J.E.; RESENDE, J.T.V.; GALVÃO, A.G.; BATTISTELLI, G.M.; MACHADO, M.M.; FARIA, M.V. Produtividade e rendimento comercial de bulbos de cebola em função da densidade de cultivo. Ciência e Agrotecnologia, v. 33, n. 10, p. 496-501, $2009 . \quad$ http://dx.doi.org/10.1590/S141370542009000200021.

BANDEIRA, G.R.L; QUEIROZ, S.O.P.; ARAGÃO, C.A.; COSTA, N.D.; SANTOS, C.A.F. Desempenho agronômico de cultivares de cebola sob diferentes manejos de irrigação no Submédio São Francisco. Irriga, v. 18, n. 1, p. 73-84, 2013. http://dx.doi.org/10.15809/

irriga.2013v18n1p73.

BOSEKENG, G.; COETZER, G.M. Response of onion (Allium cepa L.) to sowing date and plant population in the Central Free State, South Africa. African Journal Agricultural Research, v. 10, n. 4, p. 179-187, 2015. https://doi.org/10.5897/AJAR2013.8071.
COSTA, N.D.; RESENDE, G.M.; SANTOS, C.A.F.; LEITE, W.M.; PINTO, J.M. Características produtivas de genótipos de cebola no Vale do São Francisco. Horticultura Brasileira, v. 25, n. 2, p. 261264, 2007. http://dx.doi.org/10.1590/S010205362007000200026.

FERREIRA, D.F. Sisvar: A computer statistical analysis system. Ciência e Agrotecnologia v. 35, n. 6, p. 1039-1042, 2011.

GASHUA, I.B.; AHMED, A. Effect of plant population density and varietal differences on yield and yield components of onion (Allium cepa L.) in North Eastern Nigeria. International Journal Agronomy Agricultural Research, v. 3, n. 3, p. 22-27, 2013. DOI: 10.6084/M9.FIGSHARE.1455991.

GEREMEW A.; TESHOME, A.; KASAYE, T.; AMENTI, C. Effect of intra-row spacing on yield of three onion (Allium cepa L.) varieties at Adami Tulu Agricultural Research Center (Mid Rift Valley of Ethiopia). Journal Horticulture Forestry, v. 2, n. 1, p. 07-11, 2010.

HENRIQUES， G.P.S.A.; GRANGEIRO, L.C.; PAULINO, R.C.; MARROCOS, S.T.P.; SOUSA V.F.L.; RIBEIRO, R.M.P. Produção de cebola cultivada sob diferentes densidades de plantio. Revista Brasileira de Engenharia Agrícola e Ambiental, v. 18, n. 7, p. 674-679, 2014. http://dx.doi.org/10.1590/S141543662014000700002.

IBGE - INSTITUTO BRASILEIRO DE GEOGRAFIA E ESTATÍSTICA. Levantamento Sistemático da Produção Agrícola. Rio de Janeiro: IBGE. v. 30, n.11, p.1-83, 2017.

KAHSAY, Y.; BELEW, D.; ABAY, F. Intrarow spacing and variety effects on onion at Aksum, Ethiopia. African Journal 
Agricultural Research, v. 9, n. 10, p. 931 940, 2014. DOI: 10.5897/AJPS2013.1053.

LEITE, D.L.; OLIVEIRA, V.R.; SANTOS, C.A.F.; COSTA, N.D.; FONSECA, M.E.N.; BOITEUX, L.S.; MELO, P.E.; REIS, A.; UENO, B.; BAPTISTA, M.J. Melhoramento genético de cebola para as condições tropicais e subtropicais do Brasil. Revista Colombiana de Ciências Horticolas, v. 3, n. 1, p. 18 - 27, 2009. https://doi.org/10. 17584/rcch.2009v3i1.1196.

MENEZES JÚNIOR, F.O.G.; VIEIRA NETO, J. Produção da cebola em função da densidade de plantas. Horticultura Brasileira, v. 30, n. 4, p. 733 - 739, 2012. http://dx.doi.org/10. 1590/S010205362012000400028.

QUARTIERO, A.; FARIA, M.V.; RESENDE, J.T.V.; FIGUEIREDO, A.S.T.; CAMARGO, L.K.P.; SANTOS, R.L.; KOBORI, R.F. Desempenho agronômico, heterose e estabilidade fenotípica de genótipos de cebola. Horticultura Brasileira, v. 32, n. 3, p. 259-266, 2014. http://dx.doi.org/10.1590/S010205362014000300004.

REBOUÇAS, T.N.H.; SIQUEIRA, L.G.; LEMOS, O.L.; GRISI, F.A. Densidade de plantio em cebola no sistema de semeadura no Norte de Minas Gerais. Magistra, v. 20, n. 1, p. 78-86, 2008.

RESENDE, G.M.; COSTA, N.D. Produtividade e armazenamento de cebola, cv. Alfa Tropical, cultivada em diferentes espaçamentos. Horticultura Brasileira, v.
23, n. 4, p. 1010-1014, 2005a. http://dx.doi.org/10.1590/S0102-

05362005000400030.

RESENDE, G.M.; COSTA, N.D.; SANTOS, C.A.F.; SANTOS, G.M.; LEITE, W.M. Desempenho produtivo de genótipos de cebola em Vertissolo no Vale do São Francisco. Caatinga, v. 18, n. 4, p. 210-214, 2005b.

SILVA, E.; TEIXEIRA, L.A.J.; AMADO, T.J.C. The increase in onion production in Santa Catarina State, South Brasil. Onion Newsletter for the Tropics, v. 3: 7-9, 1991.

SIQUEIRA, L.G. Densidade e sistema de plantio em cebola, cultivar Serrana e híbrido Mercedes. 2004. 65 f. Dissertação (Mestrado em Produção Vegetal), UESB, Vitória da Conquista/BA.

SOUZA, J.O.; GRANGEIRO, L.C.; SANTOS, G.M.; COSTA, N.D.; SANTOS, C.A.F.; NUNES, G.H.S. Avaliação de genótipos de cebola no Semi-Árido Nordestino. Horticultura Brasileira, v. 26, n. $1, \quad$ p. $97-101,2008$. http://dx.doi.org/10.1590/S010205362008000100019.

SOUZA, R.J.; RESENDE, G.M. Cultura da cebola. Lavras: UFLA. 2002. 115p. (Textos Acadêmicos - Olericultura, 21).

TEIXEIRA, A.H.C. Informações agrometeorológicas do Pólo Petrolina, PE/Juazeiro - 1963 a 2009. Petrolina: Embrapa Semiárido, 2010. 21p. (Embrapa Semiárido. Documentos, 233). 